\title{
Diatom Species Richness in Algal Flora of Pamir, Tajikistan
}

\author{
Toirbek Niyatbekov, PhD. \\ Institute of Botany, Plant Physiology and Genetics., Dushanbe, \\ Republic of Tajikistan \\ Sophia Barinova, Prof. \\ Institute of Evolution, University of Haifa, Mount Carmel, Haifa, Israel
}

Doi: 10.19044/esj.2018.v14n3p301 URL:http://dx.doi.org/10.19044/esj.2018.v14n3p301

\begin{abstract}
The main objective of this study was to quantify the diatom floral richness and analyze the diversity structure in Pamir aquatic habitats. We revealed 455 species (552 with infraspecific taxa) of diatom algae compiled from reference studies in 1930-1983 that was done for the first time, and our data after many floristic surveys conducted in the field during 2000-2015. Floristic analysis of the total species richness revealed 65 rare and 22 species new for Pamir algal flora, and has allowed us to identify prevailed Classes, Orders, and Families in the diatoms. Only four genera are prevailing and contain about $30 \%$ of total richness. The Pinnularia species from them are representing extremely large numbers (39). They prefer fresh, clear, circumneutral-water habitats in natural aquatic objects with developed phytoperiphytonic communities, included many rare species and can be peculiarities of the Pamir diatom flora.
\end{abstract}

Keywords: Diatoms, Algal flora, Pamir, Tajikistan

\section{Introduction}

Freshwater algae are widely used in ecological assessment of water quality (Stevenson, 2014). It is very important to know about algal diversity in inland waters because most of algal species can be used as environmental indicators. Diversity of algae in Tajikistan has been studied sporadically during the last century. The uppermost, from 2,000 to 4,000 $\mathrm{m}$ above the sea level (a.s.l.), part of Tajikistan territory is Pamir where so large regional rivers as Panj and Gunt are started. This high mountain area is very rich in thermal and mineral waters, which in a way are unique habitats characterized by a constantly and high temperature from $10{ }^{\circ} \mathrm{C}$ to $86{ }^{\circ} \mathrm{C}$ and various chemical compositions saturated with carbon dioxide and nitrogen gases such as 
hydrogen sulfide-siliceous, hydrocarbonate-sulphate-calcium-magnesium, chloride-sulfate-calcium-sodium, hydrocarbonate-sulfate-sodium and weakradon-chloride-sulfate (Bokhodjaev, Davlatmamadov, 1994; Churshina, 1982). In these waters, for many centuries, a special community of algae with a specific species composition and degree of species resistance to peculiarly extreme environmental conditions was formed and developed. Therefore, Pamir is one of high altitude area in Eurasia with close relations to Hindu Cush, Altay, and Himalayas. Its territory have diverse aquatic habitats from clear freshwater large rivers, streams, lakes, to mineral and thermal springs which are occupied by diverse algal communities.

Usually, diatom algae represent about one-half of species richness in the well-studied middle-latitudinal regional algal flora. Before our study, the referenced literature data on Pamir regional algal flora is known from sporadically collected material in period 1930-1983 (Petersen, 1930; Muzafarov, 1965; Ergashev, 1969; Balashova, Nikitina, 1978; Baturina, 1983). The regular work has been started in 2000 and continued up to 2006 by G.R. Jumaeva (Jumaeva, 2008). Our own study of diatoms in thermal and mineral springs is enriched the regional diversity by 134 diatom species (166 with infraspecific taxa) (Barinova, Niyatbekov, 2017). We assume that the diversity of this group of algae in Pamir is still far from complete.

Thus, the aim of our work was to compile full taxa list of diatom algae from different habitats of Pamir from own and referenced data, and analyse their species richness and systematic structure data.

\section{Material and Methods Sampling and laboratory studies}

The material for this analysis is represented by our own data from 150 samples collected during few field trips in summer period of 2000-2015 from various thermal and mineral springs located at an altitude from 2,360 m to 3,800 m a.s.l. (Barinova, Niyatbekov, 2017). Algological samples were collected by scratching and scooping, placed in $15 \mathrm{ml}$ plastic tubes, and partly fixed with 3\% neutral formaldehyde solution, as well as partly not fixed and transported to the laboratory in the ice box.

The diatoms were prepared by the peroxide technique modified for glass slides and were placed in the Naphrax ${ }^{\circledR}$ resin from two repetitions of each sample.

The structure elements of the diatom shells were observed with Nikon stereomicroscope under magnifications $740 x-1850 x$ from two repetitions of each sample and were photographed with a DC in the Institute of Botany, Plant Physiology and Genetics, Dushanbe, Republic of Tajikistan, and the Institute of Evolution, University of Haifa, and species were defined with help of international handbooks. 


\section{Taxonomic data compilation}

Total species list was compiled from our study as well as from referenced lists in publication about diatom diversity in Pamir aquatic habitats (Petersen, 1930; Muzafarov, 1965; Ergashev, 1969; Balashova, Nikitina, 1978; Churshina, 1982; Baturina, 1983; Bokhodjaev, Davlatmamadov, 1994; Jumaeva, 2008; Barinova, Niyatbekov, 2017). All collected taxonomic data was adopted to the modern system with help of algaebase.org. Taxonomic list was analyzed in the Microsoft Access 2013 Program.

\section{Results and Discussion}

The data compilation and analysis of the floristic surveys conducted in the field has allowed us to identify 455 species (552 with infraspecific taxa) of diatom algae (Table 1). Diatom species number in the floras of surrounding mountain areas is usually contain about half of the total species richness whereas in the lowland floras the Chlorophyta species are prevail (Zarei Darki, 2009). So, diatoms are contain 498 taxa from 1,190 in South-Tajik Depression (Barinova et al., 2015), 498 taxa from 1,063 in mountain Georgia (Barinova et al., 2011), 317 taxa from 644 in the Aragvi River basin (Barinova, Kukhaleishvili, 2014), 60 from 149 taxa in Hindu Cush (Barinova et al., 2013), 690 taxa from 1,621 in Israel (Barinova, 2011), 671 taxa from 1,559 in Iran (Zarei Darki, 2009), and 961 from 2,100 in Turkey (Aysel, 2005). It let us to assume that full list of freshwater diversity in Pamir can reach about 1,000 taxa and we will continuing our study in this direction.

Table 1. Diversity of diatom algae in the Pamir high mountain habitats. Sp - species, Ssp subspecies.

\begin{tabular}{|c|c|c|c|}
\hline No. & Taxa & Sp & Ssp \\
\hline 1 & Achnanthes brevipes var. intermedia (Kützing) Cleve 1895 & 0 & 1 \\
\hline 2 & Achnanthes brevipes C.Agardh 1824 var. brevipes & 1 & 1 \\
\hline 3 & $\begin{array}{c}\text { Achnanthes coarctata (Brébisson ex W. Smith) Grunow in Cleve and } \\
\text { Grunow 1880 }\end{array}$ & 1 & 1 \\
\hline 4 & Achnanthes conspicua var. brevistriata Hustedt 1930 & 0 & 1 \\
\hline 5 & Achnanthes dispar var. angustissima (Jasnitsky) Sheshukova in \\
Proschkina-Lavrenko 1950 & 0 & 1 \\
\hline 6 & Achnanthes exigua Grunow in Cleve \& Grunow 1880 & 1 & 1 \\
\hline 7 & Achnanthes gibberula Grunow in Cleve \& Grunow 1880 var. & 1 & 1 \\
\hline 8 & gibberula & 0 & 1 \\
\hline 9 & Achnanthes gibberula var. interrupta Poretzky \& Anisimova 1933 & 1 & 1 \\
\hline 10 & Achnanthidium exile (Kützing) Heiberg 1863 & 1 & 1 \\
\hline 11 & Achnanthidium gracillimum (Meister) Lange-Bertalot 2004 & 1 & 1 \\
\hline 12 & Achnanthidium lanceolatum var. ventricosum (Hustedt) Poretsky & 0 & 1 \\
\hline
\end{tabular}




\begin{tabular}{|c|c|c|c|}
\hline 13 & Achnanthidium lineare W.Smith 1855 & 1 & 1 \\
\hline 14 & Achnanthidium minutissimum (Kützing) Czarnecki 1994 & 1 & 1 \\
\hline 15 & Achnanthidium minutum Cleve 1891 & 1 & 1 \\
\hline 16 & Achnanthidium thermale Rabenhorst 1864 & 1 & 1 \\
\hline 17 & Actinella punctata F.W. Lewis 1864 & 1 & 1 \\
\hline 18 & $\begin{array}{c}\text { Adlafia bryophila (J.B.Petersen) Lange-Bertalot in Gerd Moser, } \\
\text { Lange-Bertalot \& Metzeltin } 1998\end{array}$ & 1 & 1 \\
\hline 19 & Adlafia minuscula (Grun.) Lange-Bertalot 1999 var. minuscula & 1 & 1 \\
\hline 20 & $\begin{array}{l}\text { Adlafia minuscula var. muralis (Grunow) Lange-Bertalot in Lange- } \\
\text { Bertalot \& Genkal } 1999\end{array}$ & 0 & 1 \\
\hline 21 & Amphiprora paludosa var. duplex (Donkin) Van Heurck 1885 & 0 & 1 \\
\hline 22 & Amphora commutata Grunow in van Heurck 1880 & 1 & 1 \\
\hline 23 & Amphora libyca Ehrenberg 1841 & 1 & 1 \\
\hline 24 & Amphora mongolica Østrup 1908 var. mongolica & 1 & 1 \\
\hline 25 & Amphora mongolica var. cornuta Skvortzov 1937 & 0 & 1 \\
\hline 26 & Amphora ovalis (Kützing) Kützing 1844 var. ovalis & 1 & 1 \\
\hline 27 & Amphora ovalis var. gracilis (Ehrenberg) van Heurck 1885 & 0 & 1 \\
\hline 28 & Amphora pediculus (Kütz.) Grun. ex A. Schmidt & 1 & 1 \\
\hline 29 & Amphora proteus Gregory 1857 & 1 & 1 \\
\hline 30 & Amphora robusta Gregory 1857 & 1 & 1 \\
\hline 31 & $\begin{array}{c}\text { Aneumastus apiculatus (Østrup) Lange-Bertalot in Lange-Bertalot \& } \\
\text { Genkal } 1999\end{array}$ & 1 & 1 \\
\hline 32 & Aneumastus minor Lange-Bertalot 1993 & 1 & 1 \\
\hline 33 & Aneumastus rostratus (Hustedt) Lange-Bertalot 2001 & 1 & 1 \\
\hline 34 & $\begin{array}{c}\text { Aneumastus tuscula (Ehrenberg) D.G.Mann \& A.J.Stickle in Round, } \\
\text { R.M.Crawford \& D.G.Mann } 1990\end{array}$ & 1 & 1 \\
\hline 35 & Anomoeoneis costata (Kützing) Hustedt 1959 & 1 & 1 \\
\hline 36 & Anomoeoneis serians f. thermalis A.Cleve & 0 & 1 \\
\hline 37 & Anomoeoneis sphaerophora Pfitzer 1871 var. sphaerophora & 1 & 1 \\
\hline 38 & Anomoeoneis sphaerophora var. guentheri Otto Müller 1900 & 0 & 1 \\
\hline 39 & $\begin{array}{l}\text { Anomoeoneis sphaerophora var. sculpta (Ehrenberg) Otto Müller } \\
1900\end{array}$ & 0 & 1 \\
\hline 40 & Aulacoseira alpigena (Grunow) Krammer 1991 & 1 & 1 \\
\hline 41 & Aulacoseira distans (Ehrenberg) Simonsen 1979 & 1 & 1 \\
\hline 42 & Aulacoseira granulata (Ehrenberg) Simonsen 1979 var. granulata & 1 & 1 \\
\hline 43 & Aulacoseira granulata var. angustissima (O. Müll.) Simons. & 0 & 1 \\
\hline 44 & Aulacoseira italica (Ehrenberg) Simonsen 1979 & 1 & 1 \\
\hline 45 & $\begin{array}{l}\text { Aulacoseira nivaloides (K.E.Camburn) J.English \& M.Potapova } \\
2009\end{array}$ & 1 & 1 \\
\hline 46 & Aulacoseira valida (Grunow) Krammer 1991 & 1 & 1 \\
\hline
\end{tabular}




\begin{tabular}{|c|c|c|c|}
\hline 47 & Bacillaria paxillifera (O.F.Müller) T.Marsson 1901 & 1 & 1 \\
\hline 48 & $\begin{array}{l}\text { Boreozonacola hustedtii Lange-Bertalot, Kulikovskiy and Witkowski } \\
2010\end{array}$ & 1 & 1 \\
\hline 49 & Brachysira exilis (Kützing) Round \& D.G.Mann 1981 & 1 & 1 \\
\hline 50 & Brachysira microcephala (Grunow) Compère 1986 & 1 & 1 \\
\hline 51 & Brachysira serians (Brébisson) Round \& D.G.Mann 1981 & 1 & 1 \\
\hline 52 & Brebissonia lanceolata (Agardh) Mahoney and Reimer 1986 & 1 & 1 \\
\hline 53 & Caloneis amphisbaena (Bory) Cleve 1894 & 1 & 1 \\
\hline 54 & Caloneis bacillum (Grunow) Cleve 1894 & 1 & 1 \\
\hline 55 & Caloneis fasciata (Lagerstedt) Cleve 1894 & 1 & 1 \\
\hline 56 & Caloneis lewisii Patrick 1945 & 1 & 1 \\
\hline 57 & Caloneis limosa (Kützing) R.M.Patrick in Patrick \& Reimer 1966 & 1 & 1 \\
\hline 58 & $\begin{array}{c}\text { Caloneis molaris (Grunow) Krammer in Krammer \& Lange-Bertalot } \\
1985\end{array}$ & 1 & 1 \\
\hline 59 & Caloneis nubicola (Grunow) Cleve 1894 & 1 & 1 \\
\hline 60 & Caloneis schumaniana (Grunow) Cleve var. schumaniana & 1 & 1 \\
\hline 61 & Caloneis schumanniana var. biconstricta (Grunow) Reichelt 1903 & 0 & 1 \\
\hline 62 & Caloneis silicula (Ehrenberg) Cleve 1894 var. silicula & 1 & 1 \\
\hline 63 & Caloneis silicula var. jenissejensis Grunow & 0 & 1 \\
\hline 64 & Caloneis silicula var. kjellmaniana Cleve & 0 & 1 \\
\hline 65 & Caloneis tenuis (Gregory) Krammer 1985 & 1 & 1 \\
\hline 66 & Caloneis undulata (Gregory) Krammer 1912 & 1 & 1 \\
\hline 67 & Caloneis ventricosa (Ehrenberg) F. Meister 1912 var. ventricosa & 1 & 1 \\
\hline 68 & Caloneis ventricosa var. truncatula (Grunow) Meister 1912 & 0 & 1 \\
\hline 69 & Campylodiscus clypeus (Ehrenberg) Ehrenberg ex Kützing 1844 & 1 & 1 \\
\hline 70 & $\begin{array}{l}\text { Cavinula cocconeiformis (Gregory ex Greville) Crawford \& Mann } \\
1990\end{array}$ & 1 & 1 \\
\hline 71 & $\begin{array}{c}\text { Cavinula scutelloides (W. Smith) Lange-Bertalot in Lange-Bertalot } \\
\text { \& Metzeltin } 1996\end{array}$ & 1 & 1 \\
\hline 72 & Cocconeis disculus (Schumann) Cleve in Cleve \& Jentzsch 1882 & 1 & 1 \\
\hline 73 & Cocconeis fluviatilis J.H.Wallace 1960 & 1 & 1 \\
\hline 74 & Cocconeis neodiminuta Krammer 1990 & 1 & 1 \\
\hline 75 & Cocconeis pediculus Ehrenberg 1838 & 1 & 1 \\
\hline 76 & Cocconeis placentula Ehrenberg 1838 var. placentula & 1 & 1 \\
\hline 77 & Cocconeis placentula var. euglypta (Ehrenberg) Grunow 1884 & 0 & 1 \\
\hline 78 & $\begin{array}{c}\text { Cocconeis placentula var. intermedia (Héribaud-Joseph \& } \\
\text { M.Peragallo) Cleve } 1895\end{array}$ & 0 & 1 \\
\hline 79 & Cocconeis placentula var. lineata (Ehrenberg) Van Heurck 1885 & 0 & 1 \\
\hline 80 & $\begin{array}{l}\text { Cocconeis placentula var. rouxii (Héribaud-Joseph \& Brun) Cleve } \\
1895\end{array}$ & 0 & 1 \\
\hline
\end{tabular}




\begin{tabular}{|c|c|c|c|}
\hline 81 & Cocconeis scutellum Ehrenberg 1838 & 1 & 1 \\
\hline 82 & $\begin{array}{c}\text { Cosmioneis pusilla (W.Smith) D.G.Mann \& A.J.Stickle in Round, } \\
\text { Crawford \& Mann } 1990\end{array}$ & 1 & 1 \\
\hline 83 & $\begin{array}{c}\text { Craticula ambigua (Ehrenberg) D.G.Mann in Round, Crawford \& } \\
\text { D.G.Mann } 1990\end{array}$ & 1 & 1 \\
\hline 84 & Craticula cuspidata (Kützing) Mann 1990 & 1 & 1 \\
\hline 85 & $\begin{array}{c}\text { Craticula halophila (Grunow) D.G.Mann in Round, R.M.Crawford } \\
\text { \& D.G.Mann } 1990\end{array}$ & 1 & 1 \\
\hline 86 & $\begin{array}{l}\text { Ctenophora pulchella (Ralfs ex Kützing) D.M. Williams et Round } \\
1986 \text { var. pulchella }\end{array}$ & 1 & 1 \\
\hline 87 & Ctenophora pulchella var. lacerata (Hustedt) Bukhtiyarova 1995 & 0 & 1 \\
\hline 88 & $\begin{array}{c}\text { Cyclotella choctawhatcheeana Prasad in Prasad, Neinow \& } \\
\text { Livingston 1990: }\end{array}$ & 1 & 1 \\
\hline 89 & Cyclotella meneghiniana Kützing 1844 & 1 & 1 \\
\hline 90 & Cyclotella radiosa (Grunow) Lemmermann 1900 & 1 & 1 \\
\hline 91 & Cymatopleura elliptica (Brébisson) W.Smith 1851 & 1 & 1 \\
\hline 92 & Cymbella laevis Nägeli in Rabenhorst 1863 & 1 & 1 \\
\hline 93 & Cymbella affinis Kützing 1844 & 1 & 1 \\
\hline 94 & Cymbella amplificata Krammer 2002 & 1 & 1 \\
\hline 95 & Cymbella angustata var. diversistriata Muzafarov 1958 & 0 & 1 \\
\hline 96 & Cymbella aspera (Ehrenberg) Cleve 1894 var. aspera & 1 & 1 \\
\hline 97 & Cymbella aspera var. intermedia Skvortzov & 0 & 1 \\
\hline 98 & Cymbella cistula (Ehrenberg) O.Kirchner 1878 var. cistula & 0 & 1 \\
\hline 99 & Cymbella cistula var. maculata (Kützing) Van Heurck 1885 & 0 & 1 \\
\hline 100 & Cymbella compacta Østrup 1910 & 1 & 1 \\
\hline 101 & Cymbella cosleyi L.Bahls 2013 & 1 & 1 \\
\hline 102 & Cymbella cymbiformis C. Agardh 1830 & 1 & 1 \\
\hline 103 & Cymbella excisiformis Krammer 2002 & 1 & 1 \\
\hline 104 & Cymbella falaisensis (Grunow) Krammer \& Lange-Bertalot 1985 & 1 & 1 \\
\hline 105 & Cymbella gutwinskii (Wislouch) Skvortzov \& Meyer 1928 & 1 & 1 \\
\hline 106 & Cymbella helvetica Kützing 1844 var. helvetica & 1 & 1 \\
\hline 107 & Cymbella helvetica var. curta Cleve & 0 & 1 \\
\hline 108 & Cymbella hustedtii f. lineolata Muzafarov 1958 & 0 & 1 \\
\hline 109 & Cymbella hustedtii Krasske 1923 & 1 & 1 \\
\hline 110 & Cymbella hybrida var. pamirica J.B.Petersen & 0 & 1 \\
\hline 111 & Cymbella lanceolata (C.Agardh) C.Agardh 1830 var. lanceolata & 1 & 1 \\
\hline 112 & Cymbella lanceolata var. cornuta f. minuta Muzafarov 1958 & 0 & 1 \\
\hline 113 & Cymbella lanceolata var. notata Wisl.\& Poretzky & 0 & 1 \\
\hline 114 & Cymbella neocistula Krammer 2002 & 1 & 1 \\
\hline 115 & Cymbella obtusiuscula Kützing 1844 & 1 & 1 \\
\hline
\end{tabular}




\begin{tabular}{|c|c|c|c|}
\hline 116 & Cymbella palustris var. alpine Muzafarov 1958 & 0 & 1 \\
\hline 117 & Cymbella pamirensis Z.G.Zhang \& Rioual in Z.G.Zhang et al. 2017 & 1 & 1 \\
\hline 118 & Cymbella parva (W.Smith) Kirchner 1878 & 1 & 1 \\
\hline 119 & Cymbella proschkinae Muzafarov & 1 & 1 \\
\hline 120 & Cymbella stuxbergii (Cleve) Cleve 1894 & 1 & 1 \\
\hline 121 & Cymbella subsymmetrica J.B.Petersen & 1 & 1 \\
\hline 122 & Cymbella subturgidula Krammer 2002 & 1 & 1 \\
\hline 123 & Cymbella tropica Krammer 2002 & 1 & 1 \\
\hline 124 & Cymbella tumida (Brébisson) van Heurck 1880 & 1 & 1 \\
\hline 125 & Cymbella tumidula Grunow in Schmidt et al. 1875 & 1 & 1 \\
\hline 126 & Cymbella turgidula Grunow in A.Schmidt et al. 1875 & 1 & 1 \\
\hline 127 & Cymbella ventricosa Kützing 1844 & 1 & 1 \\
\hline 128 & Cymbella tartuensis Molder & 1 & 1 \\
\hline 129 & Cymbopleura amphicephala (Naegeli) Krammer 2003 & 1 & 1 \\
\hline 130 & Cymbopleura anglica (Lagerstedt) Krammer 2003 & 1 & 1 \\
\hline 131 & Cymbopleura angustata (W. Smith) Krammer 2003 & 1 & 1 \\
\hline 132 & Cymbopleura florentina (Grunow) K.Krammer 2003 & 1 & 1 \\
\hline 133 & Cymbopleura gutwinskii (Wislouch) Krammer 2003 & 1 & 1 \\
\hline 134 & Cymbopleura hybrida (Grunow ex Cleve) Krammer 2003 & 1 & 1 \\
\hline 135 & Cymbopleura inaequalis (Ehrenberg) Krammer 2003 & 1 & 1 \\
\hline 136 & Cymbopleura incerta (Grunow) Krammer 2003 & 1 & 1 \\
\hline 137 & Cymbopleura naviculiformis (Auerswald ex Heiberg) Krammer 2003 & 1 & 1 \\
\hline 138 & Cymbopleura reinhardtii (Grunow) K.Krammer 2003 & 1 & 1 \\
\hline 139 & Cymbopleura subaequalis (Grunov) Krammer 2003 & 1 & 1 \\
\hline 140 & Cymbopleura subcuspidata (Krammer) Krammer 2003 & 1 & 1 \\
\hline 141 & Delicata delicatula (Kützing) Krammer 2003 & 1 & 1 \\
\hline 142 & Denticula kuetzingii Grunow 1862 & 1 & 1 \\
\hline 143 & Denticula tenuis Kützing 1844 & 1 & 1 \\
\hline 144 & Denticula thermalis Kützing 1844 & 1 & 1 \\
\hline 145 & $\begin{array}{l}\text { Diadesmis contenta var. biceps (Grunow) P.B.Hamilton in Hamilton } \\
\text { et al. } 1992\end{array}$ & 0 & 1 \\
\hline 146 & Diatoma elongata (Lyngbye) C.Agardh 1824 & 1 & 1 \\
\hline 147 & Diatoma moniliformis (Kützing) D.M.Williams 2012 & 1 & 1 \\
\hline 148 & Diatoma tenuis Agardh 1812 & 1 & 1 \\
\hline 149 & Diatoma vulgaris Bory 1824 var. vulgaris & 1 & 1 \\
\hline 150 & Diatoma vulgaris var. brevis Grunow 1862 & 0 & 1 \\
\hline 151 & Diatoma vulgaris var. linearis Grunow in Van Heurck 1881 & 0 & 1 \\
\hline 152 & Diatoma vulgaris var. producta Grunow 1862 & 0 & 1 \\
\hline
\end{tabular}




\begin{tabular}{|c|c|c|c|}
\hline 153 & $\begin{array}{c}\text { Didymosphenia geminata (Lyngbye) Mart.Schmidt in A.Schmidt } \\
1899\end{array}$ & 1 & 1 \\
\hline 154 & Diploneis elliptica (Kützing) Cleve 1894 & 1 & 1 \\
\hline 155 & Diploneis oblongella (Nägeli ex Kützing) Cleve-Euler 1922 & 1 & 1 \\
\hline 156 & Diploneis oculata (Brébisson) Clve 1894 & 1 & 1 \\
\hline 157 & Diploneis ovalis (Hilse) Cleve 1891 & 1 & 1 \\
\hline 158 & Diploneis parma Cleve 1891 & 1 & 1 \\
\hline 159 & Diploneis subovalis Cleve 1894 & 1 & 1 \\
\hline 160 & Discostella stelligera (Cleve and Grunow) Houk and Klee 2004 & 1 & 1 \\
\hline 161 & Ellerbeckia arenaria (Moore ex Ralfs) Crawford 1988 & 1 & 1 \\
\hline 162 & $\begin{array}{l}\text { Encyonema alpinum (Grunow) D.G.Mann in Round, R.M.Crawford } \\
\text { \& D.G.Mann } 1990\end{array}$ & 1 & 1 \\
\hline 163 & Encyonema caespitosum Kützing 1849 & 1 & 1 \\
\hline 164 & $\begin{array}{l}\text { Encyonema elginense (Krammer) D.G.Mann in Round, Crawford \& } \\
\text { Mann } 1990\end{array}$ & 1 & 1 \\
\hline 165 & Encyonema gracile Rabenhorst 1853 & 1 & 1 \\
\hline 166 & Encyonema lacustre (C.Agardh) Pantocsek 1934 & 1 & 1 \\
\hline 167 & $\begin{array}{c}\text { Encyonema leibleinii (C.Agardh) W.J.Silva, R.Jahn, T.A.Veiga } \\
\text { Ludwig \& M.Menezes } 2013\end{array}$ & 1 & 1 \\
\hline 168 & Encyonema minutum (Hilse in Rabenhorst) D.G. Mann 1990 & 1 & 1 \\
\hline 169 & Encyonema pergracile Krammer 1997 & 1 & 1 \\
\hline 170 & Encyonema prostratum (Berkeley) Kützing 1844 & 1 & 1 \\
\hline 171 & Encyonema silesiacum (Bleisch in Rabenhorst) D.G. Mann 1990 & 1 & 1 \\
\hline 172 & Encyonopsis aequalis (W. Smith) Krammer 1997 & 1 & 1 \\
\hline 173 & Encyonopsis microcephala (Grunow) Krammer 1997 & 1 & 1 \\
\hline 174 & Entomoneis alata (Ehrenberg) Ehrenberg 1845 & 1 & 1 \\
\hline 175 & Entomoneis ornata (Bailey) Reimer 1975 & 1 & 1 \\
\hline 176 & Entomoneis paludosa (W. Smith) Reimer 1975 var. paludosa & 1 & 1 \\
\hline 177 & $\begin{array}{c}\text { Entomoneis paludosa var. duplex (Donkin) Makarova \& Achmetova } \\
1987\end{array}$ & 1 & 1 \\
\hline 178 & Epithemia adnata (Kützing) Brébisson 1838 var. adnata & 1 & 1 \\
\hline 179 & Epithemia adnata var. porcellus (Kützing) R.Ross 1950 & 0 & 1 \\
\hline 180 & $\begin{array}{c}\text { Epithemia adnata var. saxonica (Kützing) R.M.Patrick in Patrick \& } \\
\text { Reimer } 1975\end{array}$ & 0 & 1 \\
\hline 181 & Epithemia argus var. longicornis (Ehrenberg) Grunow 1862 & 0 & 1 \\
\hline 182 & Epithemia argus var. angusta Tarnavschi 1940 & 0 & 1 \\
\hline 183 & Epithemia argus (Ehrenberg) Kützing 1843 var. argus & 1 & 1 \\
\hline 184 & Epithemia argus var. alpestris (W.Smith) Grunow 1862 & 0 & 1 \\
\hline 185 & Epithemia operculata (C.Agardh) Ruck \& Nakov in Ruck et al. 2016 & 1 & 1 \\
\hline 186 & Epithemia parallela (Grunow) Ruck \& Nakov in Ruck et al. 2016 & 1 & 1 \\
\hline
\end{tabular}




\begin{tabular}{|c|c|c|c|}
\hline 187 & Epithemia sorex Kützing 1844 & 1 & 1 \\
\hline 188 & Epithemia turgida var. granulata (Ehrenberg) Brun 1880 & 0 & 1 \\
\hline 189 & Epithemia turgida (Ehrenberg) Kützing 1844 var. turgida & 1 & 1 \\
\hline 190 & Epithemia turgida var. capitata Fricke in Schmidt et al. 1904 & 0 & 1 \\
\hline 191 & Epithemia turgida var. genuina Grunow & 0 & 1 \\
\hline 192 & Eucocconeis flexella (Kützing) Meister 1912 & 1 & 1 \\
\hline 193 & Eucocconeis quadratarea (Østrup) Lange-Bertalot \& Genkal 1999 & 1 & 1 \\
\hline 194 & Eunotia pectinalis (Kützing) Rabenhorst 1864 & 1 & 1 \\
\hline 195 & Eunotia arcus Ehrenberg 1837 & 1 & 1 \\
\hline 196 & Eunotia bidens Ehrenberg 1843 & 1 & 1 \\
\hline 197 & Eunotia bilunaris (Ehrenberg) Scharschmidt 1881 & 1 & 1 \\
\hline 198 & Eunotia diodon Ehrenberg 1837 & 1 & 1 \\
\hline 199 & Eunotia exigua (Brébisson in Kützing) Rabenhorst 1864 & 1 & 1 \\
\hline 200 & Eunotia faba Ehrenberg 1837 & 1 & 1 \\
\hline 201 & Eunotia lunaris (Ehrenberg) Grunow 1877 & 1 & 1 \\
\hline 202 & Eunotia pectinalis var. minor (Kützing) Rabenhorst 1864 & 0 & 1 \\
\hline 203 & Eunotia polydentula (Brun) Hustedt 1932 & 1 & 1 \\
\hline 204 & Eunotia praerupta Ehrenberg 1843 & 1 & 1 \\
\hline 205 & Eunotia valida Hustedt 1930 & 1 & 1 \\
\hline 206 & $\begin{array}{c}\text { Fallacia insociabilis (Krasske) D.G.Mann in F.E.Round, } \\
\text { R.M.Crawford \& D.G.Mann } 1990\end{array}$ & 1 & 1 \\
\hline 207 & Fallacia pygmaea (Kützing) De Mann 1990 & 1 & 1 \\
\hline 208 & $\begin{array}{l}\text { Fragilaria acus (Kützing) Lange-Bertalot in Krammer \& Lange- } \\
\text { Bertalot } 2000\end{array}$ & 1 & 1 \\
\hline 209 & Fragilaria alpestris Krasske 1825 & 1 & 1 \\
\hline 210 & Fragilaria amphicephaloides Lange-Bertalot 2013 & 1 & 1 \\
\hline 211 & Fragilaria bicapitata A.Mayer 1917 & 1 & 1 \\
\hline 212 & Fragilaria bidens Heiberg 1863 & 1 & 1 \\
\hline 213 & Fragilaria brevistriata Grunow in Van Heurck 1885 & 1 & 1 \\
\hline 214 & Fragilaria capucina Desmazières 1830 var. capucina & 1 & 1 \\
\hline 215 & Fragilaria capucina var. lanceolata Grunow in van Heurck 1881 & 0 & 1 \\
\hline 216 & Fragilaria capucina var. vaucheriae (Kützing) Lange-Bertalot 1980 & 0 & 1 \\
\hline 217 & Fragilaria crotonensis Kitton 1869 & 1 & 1 \\
\hline 218 & Fragilaria gracilis Østrup 1910 & 1 & 1 \\
\hline 219 & Fragilaria inflata (Heiden) Hustedt 1931 var. inflata & 1 & 1 \\
\hline 220 & Fragilaria inflata var. istvanffyi (Pantoscek) Hustedt 1931 & 0 & 1 \\
\hline 221 & Fragilaria leptostauron (Ehrenberg) Hustedt 1931 & 1 & 1 \\
\hline 222 & Fragilaria mesolepta Rabenhorst 1861 & 1 & 1 \\
\hline
\end{tabular}




\begin{tabular}{|c|c|c|c|}
\hline 223 & Fragilaria radians (Kützing) Lange-Bertalot 1991 & 1 & 1 \\
\hline 224 & Fragilaria recapitellata H. Lange-Bertalot \& D. Nergui 2009 & 1 & 1 \\
\hline 225 & Fragilaria rumpens (Kützing) G.W.F.Carlson 1913 & 1 & 1 \\
\hline 226 & Fragilaria tenera (W.Smith) Lange-Bertalot 1980 & 1 & 1 \\
\hline 227 & Fragilaria vaucheriae (Kützing) J.B.Petersen 1938 & 1 & 1 \\
\hline 228 & Fragilariforma bicapitata (A.Mayer) D.M.Williams \& Round 1988 & 1 & 1 \\
\hline 229 & Fragilariforma virescens (Ralfs) D.M.Williams \& Round 1988 & 1 & 1 \\
\hline 230 & Fragilariopsis cylindrus (Grunow) Helmcke \& Krieger 1954 & 1 & 1 \\
\hline 231 & Frustulia rhomboides (Ehrenberg) De Toni 1891 & 1 & 1 \\
\hline 232 & $\begin{array}{c}\text { Genkalia digituloides (Lange-Bertalot) Lange-Bertalot \& } \\
\text { Kulikovskiy in Kulikovskiy et al. } 2012 \\
\end{array}$ & 1 & 1 \\
\hline 233 & $\begin{array}{c}\text { Gliwiczia calcar (Cleve) M.Kulikovskiy, Lange-Bertalot \& } \\
\text { A.Witkowski } 2013\end{array}$ & 1 & 1 \\
\hline 234 & Gomphoneis herculeana (Ehrenberg) Cleve 1894 & 1 & 1 \\
\hline 235 & Gomphonema acuminatum Ehrenberg 1832 & 1 & 1 \\
\hline 236 & Gomphonema constrictum var. capitatum (Ehrenberg) Grunow 1880 & 0 & 1 \\
\hline 237 & Gomphonema coronatum Ehrenberg 1840 & 1 & 1 \\
\hline 238 & Gomphonema gracile Ehrenberg 1838 & 1 & 1 \\
\hline 239 & Gomphonema parvulum (Kützing) Kützing 1849 var. parvulum & 1 & 1 \\
\hline 240 & Gomphonema parvulum var. subellipticum Cleve 1894 & 0 & 1 \\
\hline 241 & Gomphonema salinarum (Pantosek) Cleve 1894 & 1 & 1 \\
\hline 242 & Gomphonema subclavatum var. montanum (J.Schumann) Cleve & 0 & 1 \\
\hline 243 & Gomphonema ventricosum Gregory 1856 & 1 & 1 \\
\hline 244 & $\begin{array}{l}\text { Gomphonema acuminatum var. brebissonii (Kützing) Grunow in van } \\
\text { Heurck } 1880\end{array}$ & 0 & 1 \\
\hline 245 & Gomphonema angustatum (Kützing) Rabenhorst 1864 & 1 & 1 \\
\hline 246 & Gomphonema calcareum Cleve 1868 & 1 & 1 \\
\hline 247 & $\begin{array}{l}\text { Gomphonema constrictum Ehrenberg in Kützing } 1844 \text { var. } \\
\text { constrictum }\end{array}$ & 1 & 1 \\
\hline 248 & Gomphonema constrictum var. curta (Grunow) Van Heurck 1896 & 0 & 1 \\
\hline 249 & $\begin{array}{c}\text { Gomphonema exilissimum (Grunow) Lange-Bertalot \& E.Reichardt } \\
\text { in Lange-Bertalot \& Metzeltin } 1996\end{array}$ & 1 & 1 \\
\hline 250 & Gomphonema grunowii R.M.Patrick \& Reimer 1975 & 1 & 1 \\
\hline 251 & Gomphonema hedinii Hustedt 1922 & 1 & 1 \\
\hline 252 & Gomphonema intricatum Kützing 1844 & 1 & 1 \\
\hline 253 & Gomphonema kobayasii Kociolek \& J.C.Kingston 1999 & 1 & 1 \\
\hline 254 & Gomphonema lagenula Kützing 1844 & 1 & 1 \\
\hline 255 & Gomphonema longiceps Ehrenberg 1854 & 1 & 1 \\
\hline 256 & Gomphonema micropus Kützing 1844 & 1 & 1 \\
\hline 257 & Gomphonema montanum (J.Schumann) Grunow in Schneider 1878 & 1 & 1 \\
\hline
\end{tabular}




\begin{tabular}{|c|c|c|c|}
\hline 258 & Gomphonema olivaceum var. minutissimum Hustedt 1930 & 0 & 1 \\
\hline 259 & $\begin{array}{l}\text { Gomphonema olivaceum (Hornemann) Brébisson } 1838 \text { var. } \\
\text { olivaceum }\end{array}$ & 1 & 1 \\
\hline 260 & $\begin{array}{c}\text { Gomphonema productum (Grunow) Lange-Bertalot \& Reichardt in } \\
\text { Lange-Bertalot } 1993\end{array}$ & 1 & 1 \\
\hline 261 & Gomphonema subclavatum (Grunow) Grunow 1884 & 1 & 1 \\
\hline 262 & Gomphonema subsalinum Wislouch \& Poretzky in Poretzky 1924 & 1 & 1 \\
\hline 263 & Gomphonema tenellum Kützing 1844 & 1 & 1 \\
\hline 264 & Gomphonema truncatum Ehrenberg 1832 & 1 & 1 \\
\hline 265 & $\begin{array}{c}\text { Gomphonema vibrio var. pumilum (Grunow) R.Ross in B.Hartley, } \\
\text { R.Ross \& D.M.Williams 1986. }\end{array}$ & 0 & 1 \\
\hline 266 & Gomphosinica hedinii (Hustedt) Kociolek, You, Wang \& Liu 2015 & 1 & 1 \\
\hline 267 & Gyrosigma acuminatum (Kützing) Rabenhorst 1853 & 1 & 1 \\
\hline 268 & Gyrosigma attenuatum (Kützing) Rabenhorst 1894 & 1 & 1 \\
\hline 269 & Gyrosigma obtusatum (Sullivant \& Wormley) C.S.Boyer 1922 & 1 & 1 \\
\hline 270 & Gyrosigma peisone (Grunow) Hustedt in Pascher 1930 & 1 & 1 \\
\hline 271 & Gyrosigma scalproides (Rabenhorst) Cleve 1894 & 1 & 1 \\
\hline 272 & Halamphora acutiuscula (Kützing) Levkov 2009 & 1 & 1 \\
\hline 273 & Halamphora coffeaeformis (Agardh) Levkov 2009 & 1 & 1 \\
\hline 274 & Halamphora latecostata Stepanek and Kociolek 2013 & 1 & 1 \\
\hline 275 & Halamphora normanii (Rabenhorst) Levkov 2009 & 1 & 1 \\
\hline 276 & $\begin{array}{l}\text { Halamphora perpusilla (Grunow) Q.-M.You \& J.P.Kociolek in You } \\
\text { et al. } 2015\end{array}$ & 1 & 1 \\
\hline 277 & Halamphora schroederi (Hustedt) Levkov 2009 & 1 & 1 \\
\hline 278 & Halamphora subcapitata (Kisselew) Levkov 2009 & 1 & 1 \\
\hline 279 & Halamphora veneta (Kützing) Levkov 2009 & 1 & 1 \\
\hline 280 & $\begin{array}{l}\text { Hannaea arcus (Ehrenberg) Patrick } 1961 \text { emend. Genkal et } \\
\text { Kharitonov var. arcus }\end{array}$ & 1 & 1 \\
\hline 281 & Hannaea arcus var. amphioxys (Rabenhorst) R.M.Patrick 1966 & 0 & 1 \\
\hline 282 & Hannaea linearis (Holmboe) Álvarez-Blanco \& S.Blanco 2013 & 1 & 1 \\
\hline 283 & Hantzschia amphioxys (Ehr.) Grunow 1880 var. amphioxys & 1 & 1 \\
\hline 284 & Hantzschia amphioxys var. rupestris Grunow 1880 & 0 & 1 \\
\hline 285 & $\begin{array}{l}\text { Hantzschia amphioxys var. vivax (Hantzsch) Grunow in Cleve \& } \\
\text { Grunow } 1880\end{array}$ & 0 & 1 \\
\hline 286 & $\begin{array}{l}\text { Hantzschia compacta (Hustedt) Lange-Bertalot in Lange-Bertalot \& } \\
\text { Genkal } 1999\end{array}$ & 1 & 1 \\
\hline 287 & $\begin{array}{c}\text { Hippodonta capitata (Ehrenberg) Lange-Bertalot, Metzeltin \& } \\
\text { Witkowski }\end{array}$ & 1 & 1 \\
\hline 288 & $\begin{array}{c}\text { Hippodonta hungarica (Grunow) Lange-Bertalot, Metzeltin and } \\
\text { Witkowski } 1996\end{array}$ & 1 & 1 \\
\hline 289 & $\begin{array}{c}\text { Humidophila contenta (Grunow) Lowe, Kociolek, J.R.Johansen, Van } \\
\text { de Vijver, Lange-Bertalot \& Kopalová } 2014\end{array}$ & 1 & 1 \\
\hline
\end{tabular}




\begin{tabular}{|c|c|c|c|}
\hline 290 & $\begin{array}{l}\text { Humidophila perpusilla (Grunow) Lowe, Kociolek, Johansen, Van } \\
\text { de Vijver, Lange-Bertalot \& Kopalová } 2014\end{array}$ & 1 & 1 \\
\hline 291 & Iconella helvetica (Brun) Ruck \& Nakov in Ruck et al. 2016 & 1 & 1 \\
\hline 292 & Iconella linearis (W.Smith) Ruck \& Nakov in Ruck et al. 2016 & 1 & 1 \\
\hline 293 & Iconella tenera (W.Gregory) Ruck \& Nakov in Ruck et al. 2016 & 1 & 1 \\
\hline 294 & Kobayasiella micropunctata (Germain) Lange-Bertalot 1999 & 1 & 1 \\
\hline 295 & Kobayasiella subtilissima (Cleve) Lange-Bertalot 1999 & 1 & 1 \\
\hline 296 & Kurtkrammeria aequalis (W.Smith) L.Bahls 2015 & 1 & 1 \\
\hline 297 & $\begin{array}{l}\text { Lacustriella lacustris (W.Gregory) Lange-Bertalot \& } \\
\text { M.S.Kulikovskiy in Kulikovskiy et al. } 2012\end{array}$ & 1 & 1 \\
\hline 298 & Lindavia antiqua (W. Smith) Nakov et al. 2015 & 1 & 1 \\
\hline 299 & Lindavia bodanica (Eulenstein ex Grunow) T.Nakov et al. 2015 & 1 & 1 \\
\hline 300 & Lindavia comta (Kützing) T. Nakov et al. 2015 & 1 & 1 \\
\hline 301 & $\begin{array}{l}\text { Lindavia intermedia (Manguin ex Kociolek and Reviers) T. Nakov et } \\
\text { al. } 2015\end{array}$ & 1 & 1 \\
\hline 302 & $\begin{array}{c}\text { Lindavia kuetzingiana (Thwaites) T.Nakov et al. } 2015 \text { var. } \\
\text { kuetzingiana }\end{array}$ & 1 & 1 \\
\hline 303 & Lindavia kuetzingiana var. radiosa (Fricke) T.Nakov et al. 2015 & 1 & 1 \\
\hline 304 & Lindavia lacunarum (Hustedt) T. Nakov et al. 2015 & 1 & 1 \\
\hline 305 & Lindavia schumannii (Grunow) T.Nakov et al. 2015 & 1 & 1 \\
\hline 306 & $\begin{array}{c}\text { Luticola cohnii (Hilse) D.G.Mann in Round, R.M.Crawford \& } \\
\text { D.G.Mann } 1990\end{array}$ & 1 & 1 \\
\hline 307 & Luticola mutica (Kützing) D.G.Mann in Round et al. 1990 & 1 & 1 \\
\hline 308 & $\begin{array}{l}\text { Luticola muticopsis (Van Heurck) D.G.Mann in Round, } \\
\text { R.M.Crawford \& D.G.Mann } 1990\end{array}$ & 1 & 1 \\
\hline 309 & $\begin{array}{c}\text { Luticola nivalis (Ehrenberg) D.G.Mann in Round, R.M.Crawford \& } \\
\text { D.G.Mann } 1990\end{array}$ & 1 & 1 \\
\hline 310 & $\begin{array}{c}\text { Mastogloia albertii A.Pavlov, E.Jovanovska, C.E.Wetzel, L.Ector \& } \\
\text { Z.Levkov } 2016\end{array}$ & 1 & 1 \\
\hline 311 & Mastogloia baltica Grunow in van Heurck 1880 & 1 & 1 \\
\hline 312 & Mastogloia braunii Grunow 1863 & 1 & 1 \\
\hline 313 & Mastogloia elliptica (C.Agardh) Cleve in Schmidt et al. 1893 & 1 & 1 \\
\hline 314 & Mastogloia lacustris (Grunow) Grunow in Van Heurck 1880 & 1 & 1 \\
\hline 315 & $\begin{array}{c}\text { Mastogloia pseudosmithii S.S.Lee, E.E.Gaiser, B.Van de Vijver, } \\
\text { M.B.Edlund \& Spaulding } 2014\end{array}$ & 1 & 1 \\
\hline 316 & Mastogloia pumila (Grunow) Cleve 1895 & 1 & 1 \\
\hline 317 & Mastogloia smithii Thwaites 1856 & 1 & 1 \\
\hline 318 & Meridion circulare (Greville) Agardh 1831 & 1 & 1 \\
\hline 319 & Meridion constrictum Ralfs 1843 & 1 & 1 \\
\hline 320 & Meridion lineare Williams 1985 & 1 & 1 \\
\hline 321 & Navicula brasiliensis Grunow 1863 & 1 & 1 \\
\hline 322 & Navicula bryophila Østrup & 1 & 1 \\
\hline
\end{tabular}




\begin{tabular}{|c|c|c|c|}
\hline 323 & Navicula capitatoradiata H.Germain 1981 & 1 & 1 \\
\hline 324 & Navicula cari Ehrenberg 1836 & 1 & 1 \\
\hline 325 & Navicula cincta (Ehrenberg) Ralfs 1861 & 1 & 1 \\
\hline 326 & Navicula cryptocephala Kützing 1844 var. cryptocephala & 1 & 1 \\
\hline 327 & Navicula cryptocephala var. lata Poretz. et Anissimova 1933 & 0 & 1 \\
\hline 328 & Navicula dicephala Ehrenberg 1838 var. dicephala & 1 & 1 \\
\hline 329 & Navicula dicephala var. triundulata Kisselev 1937 & 0 & 1 \\
\hline 330 & Navicula digitoradiata (Gregory) Ralfs in Prichard 1861 & 1 & 1 \\
\hline 331 & Navicula exilis Kützing 1844 & 1 & 1 \\
\hline 332 & Navicula fritschii J.W.G.Lund 1946 & 1 & 1 \\
\hline 333 & Navicula gothlandica Grunow in Van Heurck 1880 & 1 & 1 \\
\hline 334 & Navicula gregaria Donkin 1861 & 1 & 1 \\
\hline 335 & Navicula halophila f. subcapitata (Østrup) Krasske 1929 & 0 & 1 \\
\hline 336 & Navicula hofmanniae Lange-Bertalot 1993 & 1 & 1 \\
\hline 337 & Navicula lacustris var. parallela Wisl. \& Kolbe 1916 & 0 & 1 \\
\hline 338 & Navicula lacustris var. paulseniana (J.B.Petersen) Zabelina 1951 & 0 & 1 \\
\hline 339 & Navicula lanceolata (Agardh) Ehrenberg 1838 & 1 & 1 \\
\hline 340 & Navicula libonensis Schoeman 1970 & 1 & 1 \\
\hline 341 & Navicula lucidula Grunow in Van Heurck 1880 & 1 & 1 \\
\hline 342 & Navicula menisculus Schumann 1867 & 1 & 1 \\
\hline 343 & Navicula microstauron f. diminuta Grunow & 0 & 1 \\
\hline 344 & Navicula minima Grunow in van Heurck 1880 & 1 & 1 \\
\hline 345 & Navicula oblonga (Kützing) Kützing 1844 & 1 & 1 \\
\hline 346 & Navicula peregrina (Ehrenberg) Kützing 1843 & 1 & 1 \\
\hline 347 & Navicula pygmaea (Kützing) Pantocsek 1901 & 1 & 1 \\
\hline 348 & Navicula radiosa Kützing 1844 & 1 & 1 \\
\hline 349 & $\begin{array}{c}\text { Navicula recens (Lange-Bertalot) Lange-Bertalot in Krammer \& } \\
\text { Lange-Bertalot } 1985\end{array}$ & 1 & 1 \\
\hline 350 & Navicula reinhardtii Grun. & 1 & 1 \\
\hline 351 & Navicula rhynchocephala Ehrenberg 1844 & 1 & 1 \\
\hline 352 & Navicula rostellata Kützing 1844 & 1 & 1 \\
\hline 353 & Navicula rotaeana (Rabenhorst) Grunow in van Heurck 1880 & 1 & 1 \\
\hline 354 & Navicula salinarum f. minima Kolbe 1927 & 0 & 1 \\
\hline 355 & Navicula scutum Schumann 1862 & 1 & 1 \\
\hline 356 & Navicula semen Ehrenberg 1843 & 1 & 1 \\
\hline 357 & Navicula tenella Brébisson ex Kützing 1849 & 1 & 1 \\
\hline 358 & $\begin{array}{l}\text { Navicula tripunctata (O.F.Müller) Bory in Bory de Saint-Vincent } \\
1822\end{array}$ & 1 & 1 \\
\hline
\end{tabular}




\begin{tabular}{|c|c|c|c|}
\hline 359 & Navicula tuscula f. intermedia Kisselev 1932 & 0 & 1 \\
\hline 360 & Navicula veneta Kützing 1844 & 1 & 1 \\
\hline 361 & Navicula viridula (Kützing) Ehrenberg 1836 & 1 & 1 \\
\hline 362 & Navicula vulpina Kützing 1844 & 1 & 1 \\
\hline 363 & Navicymbula pusilla (Grunow in A. Schmidt) Krammer 2003 & 1 & 1 \\
\hline 364 & $\begin{array}{c}\text { Neidiomorpha binodis (Ehrenberg) M.Cantonati, Lange-Bertalot \& } \\
\text { N.Angeli } 2010\end{array}$ & 1 & 1 \\
\hline 365 & Neidium affine (Ehrenberg) Pfizer 1871 f. affine & 1 & 1 \\
\hline 366 & Neidium affine f. undulatum (Grunow) Hustedt 1930 & 0 & 1 \\
\hline 367 & Neidium affine var. amphirhynchus (Ehrenberg) Cleve 1894 & 0 & 1 \\
\hline 368 & Neidium iridis (Ehrenberg) Cleve 1894 & 1 & 1 \\
\hline 369 & Neidium kozlowii Mereschkovsky 1906 & 1 & 1 \\
\hline 370 & Neidium productum (W.Smith) Cleve 1894 & 1 & 1 \\
\hline 371 & Neidium punctulatum Hustedt & 1 & 1 \\
\hline 372 & Neidium undulatum Bahls 2013 & 1 & 1 \\
\hline 373 & Nitzschia ostenfeldii Hustedt & 1 & 1 \\
\hline 374 & Nitzschia commutata Grunow in Cleve \& Grunow 1880 & 1 & 1 \\
\hline 375 & Nitzschia amphibia Grunow 1862 var. amphibia & 1 & 1 \\
\hline 376 & Nitzschia amphibia var. thermalis Grunow 1862 & 0 & 1 \\
\hline 377 & Nitzschia angularis W.Smith 1853 & 1 & 1 \\
\hline 378 & Nitzschia angustata var. acuta Grunow in Cleve \& Grunow 1880 & 0 & 1 \\
\hline 379 & Nitzschia brevissima Grunow in Van Heurck 1880 & 1 & 1 \\
\hline 380 & Nitzschia communis Rabenhorst 1860 & 1 & 1 \\
\hline 381 & Nitzschia denticula Grunow in Cleve \& Grunow 1880 & 1 & 1 \\
\hline 382 & Nitzschia dissipata (Kützing) Rabenhorst 1860 & 1 & 1 \\
\hline 383 & Nitzschia distans W.Gregory 1857 & 1 & 1 \\
\hline 384 & Nitzschia dubia W.Smith 1853 & 1 & 1 \\
\hline 385 & Nitzschia fasciculata (Grunow) Grunow in Van Heurck 1881 & 1 & 1 \\
\hline 386 & Nitzschia fonticola (Grunow) Grunow in Van Heurck 1881 & 1 & 1 \\
\hline 387 & $\begin{array}{l}\text { Nitzschia frustulum (Kützing) Grunow in Cleve \& Grunow } 1880 \text { var. } \\
\text { frustulum }\end{array}$ & 1 & 1 \\
\hline 388 & Nitzschia frustulum var. subsalina Hustedt 1930 & 0 & 1 \\
\hline 389 & Nitzschia frustulum var. perpusilla (Rabenhorst) Van Heurck 1885 & 0 & 1 \\
\hline 390 & Nitzschia gracilis Hantzsch1860 & 1 & 1 \\
\hline 391 & Nitzschia gradifera Hustedt 1922 & 1 & 1 \\
\hline 392 & Nitzschia hantzschiana Rabenhorst 1860 & 1 & 1 \\
\hline 393 & Nitzschia heidenii (Meister) Hustedt 1924 var. heidenii & 1 & 1 \\
\hline 394 & Nitzschia heidenii var. pamirensis J.B.Petersen 1930 & 0 & 1 \\
\hline
\end{tabular}




\begin{tabular}{|c|c|c|c|}
\hline 395 & Nitzschia holsatica Hustedt 1930 & 1 & 1 \\
\hline 396 & Nitzschia hungarica var. pantocsekii Wisl.\& Poretzky & 0 & 1 \\
\hline 397 & Nitzschia kittlii Grunow 1882 & 1 & 1 \\
\hline 398 & Nitzschia kuetzingiana Hilse 1863 & 1 & 1 \\
\hline 399 & $\begin{array}{c}\text { Nitzschia linearis var. tenuis (W.Smith) Grunow in Cleve \& Grunow } \\
1880\end{array}$ & 0 & 1 \\
\hline 400 & Nitzschia linearis W.Smith 1853 var. linearis & 1 & 1 \\
\hline 401 & Nitzschia microcephala Grunow in Cleve \& Möller 1878 & 1 & 1 \\
\hline 402 & Nitzschia obtusa W.Smith 1853 & 1 & 1 \\
\hline 403 & Nitzschia palea (Kützing) W. Smith 1856 & 1 & 1 \\
\hline 404 & Nitzschia paleaceae Grunow in Van Heurck 1881 & 1 & 1 \\
\hline 405 & Nitzschia parvula W.Smith 1853 & 1 & 1 \\
\hline 406 & Nitzschia regula Hustedt var. regula & 1 & 1 \\
\hline 407 & Nitzschia regula var. robusta Hustedt 1924 & 0 & 1 \\
\hline 408 & Nitzschia sigma (Kützing) W. Smith 1853 var. sigma & 1 & 1 \\
\hline 409 & Nitzschia sigma var. curvula (Ehrenberg) Brun. & 0 & 1 \\
\hline 410 & Nitzschia sigmoidea (Nitzsch) W.Smith 1853 & 1 & 1 \\
\hline 411 & Nitzschia solgensis Cleve-Euler 1952 & 1 & 1 \\
\hline 412 & Nitzschia sublinearis Hustedt 1930 & 1 & 1 \\
\hline 413 & Nitzschia subtilis (Kützing) Grunow in Cleve \& Grunow 1880 & 1 & 1 \\
\hline 414 & Nitzschia supralitorea Lange-Bertalot & 1 & 1 \\
\hline 415 & $\begin{array}{l}\text { Nitzschia thermalis (Ehrenberg) Auerswald in Rabenhorst } 1861 \text { var. } \\
\text { thermalis }\end{array}$ & 1 & 1 \\
\hline 416 & Nitzschia thermalis var. minor Hilse 1862 & 0 & 1 \\
\hline 417 & Nitzschia tryblionella Hantzsch in Rabenhorst 1860 & 1 & 1 \\
\hline 418 & Nitzschia vermicularis (Kützing) Hantzsch in Rabenhorst 1860 & 1 & 1 \\
\hline 419 & Odontidium mesodon (Kützing) Kützing 1849 & 1 & 1 \\
\hline 420 & $\begin{array}{l}\text { Paraplaconeis compositestriata (Jasnitsky) Kulikovskiy, Metzeltin \& } \\
\text { Lange-Bertalot in Kulikovskiy et al. } 2012\end{array}$ & 1 & 1 \\
\hline 421 & $\begin{array}{c}\text { Paraplaconeis placentula (Ehrenberg) M.S.Kulikovskiy \& Lange- } \\
\text { Bertalot in Kulikowskiy et al. } 2012\end{array}$ & 1 & 1 \\
\hline 422 & $\begin{array}{c}\text { Paraplaconeis subplacentula (Hustedt) Kulikovskiy \& Lange- } \\
\text { Bertalot in Kulikovskiy et al. } 2012\end{array}$ & 1 & 1 \\
\hline 423 & $\begin{array}{c}\text { Parlibellus crucicula (W.Smith) Witkowski, Lange-Bertalot \& } \\
\text { Metzeltin } 2000\end{array}$ & 1 & 1 \\
\hline 424 & $\begin{array}{c}\text { Parlibellus protracta (Grunow) Witkowski, Lange-Bertalot \& } \\
\text { Metzeltin } 2000\end{array}$ & 1 & 1 \\
\hline 425 & Pinnularia abaujensis var. linearis (Hustedt) R.M.Patrick 1966 & 0 & 1 \\
\hline 426 & Pinnularia acrosphaeria W. Smith 1853 & 1 & 1 \\
\hline 427 & Pinnularia appendiculata (C.Agardh) Schaarschmidt 1881 & 1 & 1 \\
\hline 428 & Pinnularia biceps W.Gregory 1856 & 1 & 1 \\
\hline
\end{tabular}




\begin{tabular}{|c|c|c|c|}
\hline 429 & Pinnularia borealis Ehrenberg 1843 & 1 & 1 \\
\hline 430 & Pinnularia brauniana (Grunow) Studnicka 1888 & 1 & 1 \\
\hline 431 & Pinnularia brebissonii (Kützing) Rabenhorst 1864 & 1 & 1 \\
\hline 432 & Pinnularia brevicostata Cleve 1891 & 1 & 1 \\
\hline 433 & $\begin{array}{c}\text { Pinnularia canadodivergens Kulikovsky, Lange-Bertalot et } \\
\text { Metzeltin } 2010\end{array}$ & 1 & 1 \\
\hline 434 & Pinnularia divergens W.Smith 1853 & 1 & 1 \\
\hline 435 & Pinnularia divergentissima (Grunow) Cleve 1895 & 1 & 1 \\
\hline 436 & Pinnularia elegans (W.Smith) K.Krammer 1992 & 1 & 1 \\
\hline 437 & Pinnularia fonticola Hustedt 1922 & 1 & 1 \\
\hline 438 & Pinnularia gibbiformis K.Krammer 1992 & 1 & 1 \\
\hline 439 & Pinnularia globiceps W.Gregory 1856 & 1 & 1 \\
\hline 440 & Pinnularia gracillima W.Gregory 1856 & 1 & 1 \\
\hline 441 & Pinnularia grunowii Krammer 2000 & 1 & 1 \\
\hline 442 & Pinnularia hartleyana Greville 1865 & 1 & 1 \\
\hline 443 & Pinnularia interrupta W.Smith 1853 & 1 & 1 \\
\hline 444 & Pinnularia lata (Brébisson) W.Smith 1853 var. lata & 1 & 1 \\
\hline 445 & Pinnularia lata var. minor (Grunow) Cleve & 0 & 1 \\
\hline 446 & Pinnularia major (Kützing) Rabenhorst 1853 & 1 & 1 \\
\hline 447 & Pinnularia mesolepta (Ehrenberg) W.Smith 1853 & 1 & 1 \\
\hline 448 & Pinnularia microstauron (Ehrenberg) Cleve 1891 & 1 & 1 \\
\hline 449 & Pinnularia rangoonensis Grunow ex Cleve 1895 & 1 & 1 \\
\hline 450 & Pinnularia rhombarea var. biundulata (Otto Müller) Krammer 2000 & 0 & 1 \\
\hline 451 & Pinnularia rhombica Hustedt in Schmidt et al. 1934 & 1 & 1 \\
\hline 452 & Pinnularia schoenfelderi Krammer 1992 & 1 & 1 \\
\hline 453 & Pinnularia septentrionalis K.Krammer 2000 & 1 & 1 \\
\hline 454 & Pinnularia socialis var. debesii (Hustedt) Krammer 2000 & 0 & 1 \\
\hline 455 & Pinnularia stomatophora Hustedt 1935 & 1 & 1 \\
\hline 456 & Pinnularia subborealis Hustedt 1922 & 1 & 1 \\
\hline 457 & Pinnularia subtibetana A.M.Muzafarov & 1 & 1 \\
\hline 458 & Pinnularia tabellaria Ehrenberg 1843 var. tabellaria & 1 & 1 \\
\hline 459 & Pinnularia tabellaria var. stauroneiformis (van Heurck) Boyer 1927 & 0 & 1 \\
\hline 460 & Pinnularia undulata W.Gregory 1854 & 1 & 1 \\
\hline 461 & Pinnularia viridiformis Krammer 1992 & 1 & 1 \\
\hline 462 & Pinnularia viridis (Nitzsch) Ehrenberg 1843 var. viridis & 1 & 1 \\
\hline 463 & Pinnularia viridis var. diminuta Mayer 1913 & 0 & 1 \\
\hline 464 & Placoneis amphibola (Cleve) E.J.Cox 2003 & 1 & 1 \\
\hline 465 & Placoneis exigua (Gregory) Mereschkovsky & 1 & 1 \\
\hline
\end{tabular}




\begin{tabular}{|c|c|c|c|}
\hline 466 & Placoneis gastrum (Ehrenberg) Mereschkowsky 1903 & 1 & 1 \\
\hline 467 & $\begin{array}{l}\text { Planothidium dispar (Cleve) Witkowski, Lange-Bertalot \& Metzeltin } \\
\qquad 2000\end{array}$ & 1 & 1 \\
\hline 468 & $\begin{array}{l}\text { Planothidium lanceolatum (Brébisson ex Kützing) Lange-Bertalot } \\
1999\end{array}$ & 1 & 1 \\
\hline 469 & $\begin{array}{c}\text { Planothidium rostratoholoarcticum Lange-Bertalot \& Bąk in B?k \& } \\
\text { Lange-Bertalot } 2015\end{array}$ & 1 & 1 \\
\hline 470 & Planothidium rostratum (Østrup) Lange-Bertalot 1999 & 1 & 1 \\
\hline 471 & Platessa salinarum (Grunow) Lange-Bertalot & 1 & 1 \\
\hline 472 & Prestauroneis integra (W. Smith) Bruder 2008 & 1 & 1 \\
\hline 473 & $\begin{array}{c}\text { Prestauroneis protracta (Grunow) I.W.Bishop, Minerovic, Q.Liu \& } \\
\text { Kociolek in I.W.Bishop, Minerovic \& Kociolek } 2017\end{array}$ & 1 & 1 \\
\hline 474 & Pseudostaurosira brevistriata (Grunow) Williams and Round 1987 & 1 & 1 \\
\hline 475 & $\begin{array}{l}\text { Pseudostaurosira elliptica (Schumann) Edlund, Morales \& } \\
\text { Spaulding } 2006\end{array}$ & 1 & 1 \\
\hline 476 & Pseudostaurosira parasitica (W.Smith) Morales 2003 & 1 & 1 \\
\hline 477 & Pseudostaurosira robusta (Fusey) Williams and Round 1988 & 1 & 1 \\
\hline 478 & Pseudostaurosira subsalina (Hustedt) E.A. Morales 2005 & 1 & 1 \\
\hline 479 & $\begin{array}{l}\text { Pseudostaurosira pseudoconstruens (Marciniak) Williams and } \\
\text { Round } 1987\end{array}$ & 1 & 1 \\
\hline 480 & $\begin{array}{l}\text { Punctastriata lancettula (Schumann) P.B.Hamilton \& P.A.Siver } \\
2008\end{array}$ & 1 & 1 \\
\hline 481 & Reimeria sinuata (Greg.) Kociolek et Stoermer & 1 & 1 \\
\hline 482 & Rexlowea navicularis Kociolek and Thomas 2010 & 1 & 1 \\
\hline 483 & Rhoicosphenia abbreviata (Agardh) Lange-Bertalot 1980 & 1 & 1 \\
\hline 484 & Rhopalodia gibba (Ehrenberg) Otto Müller 1895 var. gibba & 1 & 1 \\
\hline 485 & $\begin{array}{c}\text { Rhopalodia gibba var. mongolica (Østrup) Proschkina-Lavrenko } \\
1950\end{array}$ & 0 & 1 \\
\hline 486 & Rhopalodia gibba var. ventricosa (Kützing) Mayer 1913 & 0 & 1 \\
\hline 487 & Rhopalodia gibberula (Ehrenberg) O. Müller 1895 var. gibberula & 1 & 1 \\
\hline 488 & Rhopalodia gibberula var. producta (Grunow) Otto Müller 1900 & 0 & 1 \\
\hline 489 & Rhopalodia musculus (Kützing) O. Müller 1900 var. musculus & 1 & 1 \\
\hline 490 & Rhopalodia musculus var. mirabilis Fricke & 0 & 1 \\
\hline 491 & Rossithidium anastasiae (Kaczmarska) Potapova 2012 & 1 & 1 \\
\hline 492 & Sellaphora bacillum (Ehrenberg) D.G. Mann 1989 & 1 & 1 \\
\hline 493 & Sellaphora pupula (Kützing) Mereschkowsky 1902 & 1 & 1 \\
\hline 494 & $\begin{array}{c}\text { Sellaphora rectangularis (W.Gregory) Lange-Bertalot \& Metzeltin } \\
1996\end{array}$ & 1 & 1 \\
\hline 495 & Sellaphora tridentula (Krasske) C.E.Wetzel in Wetzel et al. 2015 & 1 & 1 \\
\hline 496 & Stauroneis borrichii (J.B.Petersen) J.W.G.Lund 1946 & 1 & 1 \\
\hline 497 & Stauroneis acuta W. Smith 1853 & 1 & 1 \\
\hline 498 & Stauroneis anceps Ehrenberg 1843 var. anceps & 1 & 1 \\
\hline
\end{tabular}




\begin{tabular}{|c|c|c|c|}
\hline 499 & Stauroneis anceps var. argentina (Cleve) Cleve & 0 & 1 \\
\hline 500 & Stauroneis borrichi var. subcapitata (Peterson) Hustedt 195 & 0 & 1 \\
\hline 501 & Stauroneis gracilis Ehrenberg 1843 & 1 & 1 \\
\hline 502 & Stauroneis lauenburgiana Hustedt 1950 & 1 & 1 \\
\hline 503 & Stauroneis phoenicenteron (Nitzsch) Ehrenberg 1843 & 1 & 1 \\
\hline 504 & Stauroneis smithii Grunow 1860 & 1 & 1 \\
\hline 505 & Staurosira binodis (Ehrenberg) Lange-Bertalot 2011 & 1 & 1 \\
\hline 506 & Staurosira construens Ehrenberg 1843 var. construens & 1 & 1 \\
\hline 507 & Staurosira construens var. exigua (W.Smith) T.Nagumo 2002 & 0 & 1 \\
\hline 508 & Staurosira venter (Ehrenberg) Cleve \& J.D.Möller 1879 & 1 & 1 \\
\hline 509 & $\begin{array}{l}\text { Staurosirella harrisonii (W.Smith) E. Morales \& C.E.Wetzel in } \\
\text { Morales et al. } 2015\end{array}$ & 1 & 1 \\
\hline 510 & Staurosirella leptostauron (Ehrenberg) Williams and Round 1987 & 1 & 1 \\
\hline 511 & Staurosirella pinnata (Ehrenberg) Williams and Round 1987 & 1 & 1 \\
\hline 512 & $\begin{array}{l}\text { Stephanodiscus astraea (Ehrenberg) Grunow in Cleve \& Grunow } \\
1880 \text { var. astraea }\end{array}$ & 1 & 1 \\
\hline 513 & Stephanodiscus astraea var. intermedia Fricke & 1 & 1 \\
\hline 514 & Surirella angusta Kützing 1844 & 1 & 1 \\
\hline 515 & Surirella angustata Hustedt 1930 var. angustata & 1 & 1 \\
\hline 516 & Surirella angustata var. constricta Hustedt & 0 & 1 \\
\hline 517 & Surirella brebissonii Krammer and Lange-Bertalot 1987 & 1 & 1 \\
\hline 518 & Surirella librile (Ehrenberg) Ehrenberg 1845 & 1 & 1 \\
\hline 519 & Surirella minuta Brébisson in Kützing 1849 & 1 & 1 \\
\hline 520 & Surirella ovalis Brébisson 1838 & 1 & 1 \\
\hline 521 & Surirella ovata var. crumena (Brébisson) Hustedt 1930 & 0 & 1 \\
\hline 522 & Surirella spiralis Kützing 1844 & 1 & 1 \\
\hline 523 & Surirella splendida (Ehrenberg) Kützing 1844 & 1 & 1 \\
\hline 524 & Synedra acus var. radians (Kützing) Hustedt 1930 & 0 & 1 \\
\hline 525 & Synedra famelica Kützing 1844 & 1 & 1 \\
\hline 526 & Synedra goulardii Brébisson ex Cleve and Grunow 1880 & 1 & 1 \\
\hline 527 & Synedra minuscula Grunow in Van Heurck 1881 & 1 & 1 \\
\hline 528 & Synedra montana Krasske ex Hustedt 1932 & 1 & 1 \\
\hline 529 & Synedra nana F.Meister 1912 & 1 & 1 \\
\hline 530 & Synedra parasitica (W.Smith) Hustedt 1930 & 1 & 1 \\
\hline 531 & Synedra pulchella var. macrocephala Grunow 1880 & 0 & 1 \\
\hline 532 & Synedra spectabilis Ehrenberg 1841 & 1 & 1 \\
\hline 533 & Tabellaria fenestrata (Lyngbye) Kützing 1844 & 1 & 1 \\
\hline 534 & Tabellaria flocculosa (Roth) Kützing 1844 & 1 & 1 \\
\hline
\end{tabular}




\begin{tabular}{|l|c|c|c|}
\hline 535 & Tabularia fasciculata (C.Agardh) D.M.Williams \& Round 1986 & 1 & 1 \\
\hline 536 & Tetracyclus rupestris (Kützing) Grunow in Van Heurck 1881 & 1 & 1 \\
\hline 537 & Tryblionella angustata W.Smith 1853 & 1 & 1 \\
\hline 538 & Tryblionella apiculata W.Gregory 1857 & 1 & 1 \\
\hline 539 & Tryblionella hantzschiana Grunow & 1 & 1 \\
\hline 540 & Tryblionella hungarica (Grunow) Frenguelli 1942 & 1 & 1 \\
\hline 541 & Tryblionella levidensis W.Smith 1856 & 1 & 1 \\
\hline 542 & Tryblionella victoriae Grunow 1862 & 1 & 1 \\
\hline 543 & Ulnaria acus Aboal 2003 & 1 & 1 \\
\hline 544 & Ulnaria amphirhynchus (Ehrenberg) Compère \& Bukhtiyarova 2006 & 1 & 1 \\
\hline 545 & Ulnaria biceps (Kützing) Compére 2001 & 1 & 1 \\
\hline 546 & Ulnaria capitata (Ehrenberg) Compère 2001 & 1 & 1 \\
\hline 547 & Ulnaria contracta (Østrup) E.A.Morales \& M.L.Vis 2007 & 1 & 1 \\
\hline 548 & Ulnaria danica (Kützing) Compère \& Bukhtiyarova in Bukhtiyarova & 1 & 1 \\
\hline 549 & \& Compère 2006 & 0 & 1 \\
\hline 550 & Ulnaria delicatissima var. angustissima (Grun.) Aboal et Silva & 1 & 1 \\
\hline 551 & oxyrhynchus (Kützing) Aboal in Aboal, Alvarez Cobelas, & 1 & 1 \\
\hline 552 & Cambra \& Ector 2003 & 1 & 1 \\
\hline & Ulnaria ulna var. aequalis (Kützing) Aboal in Aboal, Alvarez & 0 & 55 \\
\hline
\end{tabular}

The study of the taxonomic structure of the flora is an important part of the floristic analysis, which makes it possible to identify the most diversified taxa of the floristic spectrum, showing the direction of the algal flora development of the region as a whole.

The diatoms found are subdivided into three classes: Bacillariophyceae, Coscinodiscophyceae, Mediophyceae, and two species has unclear position (Table 2). Most richest is Class Bacillariophyceae, included $94.97 \%$ of species. Order Naviculales prevail with $30.44 \%$ of total richness with Families Naviculaceae (12.01\%) and Pinnulariaceae (7.12\%) in leading position. One of the important characteristics of the flora is the composition of the leading algae genera. So, in Pamir diatom flora only four genera contain $30 \%$ of the list. It is Nitzschia (45 taxa), Navicula (41 taxa), Pinnularia (39 taxa), and Cymbella (36 taxa). Whereas three genera of them is usually are in the head part of the diatom flora list, it looks like surprisingly so large number of Pinnularia members can be describe as pecularities of Pamir diatom flora. Ecologically this are the species preferred fresh, clear, circumneutral water habitats in natural aquatic objects with developed phytoperiphytonic communities and included many rare species. 
Table 2. Systematical structure of diatom algal diversity in the Pamir high mountain habitats. Note: incertae sedis unclear taxonomical position.

\begin{tabular}{|c|c|c|c|c|}
\hline Taxa & Genera & Species & Subspecies & Percent \\
\hline Division Bacillariophyta & 93 & 455 & 552 & 100.00 \\
\hline Class 1. Bacillariophyceae & 86 & 437 & 539 & 94.67 \\
\hline Order 1. Bacillariales & 6 & 51 & 65 & 11.54 \\
\hline Family 1. Bacillariaceae & 6 & 51 & 65 & 11.54 \\
\hline Order 2. Cocconeidales & 5 & 19 & 24 & 4.26 \\
\hline Family 2. Achnanthidiaceae & 4 & 13 & 14 & 2.48 \\
\hline Family 3. Cocconeidaceae & 1 & 6 & 10 & 1.78 \\
\hline Order 3. Cymbellales & 17 & 93 & 114 & 19.3 \\
\hline Family 4. Anomoeoneidaceae & 2 & 4 & 8 & 1.43 \\
\hline Family 5. Cymbellaceae & 6 & 45 & 55 & 9.61 \\
\hline Family 6. Gomphonemataceae & 8 & 43 & 50 & 8.09 \\
\hline Family 7. Rhoicospheniaceae & 1 & 1 & 1 & 0.17 \\
\hline Order 4. Eunotiales & 2 & 13 & 14 & 2.48 \\
\hline Family 8. Eunotiaceae & 2 & 13 & 14 & 2.48 \\
\hline Order 5. Fragilariales & 8 & 41 & 47 & 8.35 \\
\hline Family 9. Fragilariaceae & 5 & 29 & 34 & 6.03 \\
\hline Family 10. Staurosiraceae & 3 & 12 & 13 & 2.32 \\
\hline Order 6. Licmophorales & 4 & 12 & 16 & 2.84 \\
\hline Family 11. Ulnariaceae & 4 & 12 & 16 & 2.84 \\
\hline Order 7. Mastogloiales & 4 & 18 & 22 & 3.91 \\
\hline Family 12. Achnanthaceae & 2 & 6 & 10 & 1.78 \\
\hline Family 13. Mastogloiaceae & 2 & 12 & 12 & 2.13 \\
\hline Order 8. Naviculales & 28 & 147 & 172 & 30.44 \\
\hline Family 14. Amphipleuraceae & 3 & 9 & 10 & 1.77 \\
\hline Family 15. Berkeleyaceae & 1 & 2 & 2 & 0.35 \\
\hline Family 16. Brachysiraceae & 1 & 3 & 3 & 0.53 \\
\hline Family 17. Cavinulaceae & 1 & 2 & 2 & 0.35 \\
\hline Family 18. Cosmioneidaceae & 1 & 1 & 1 & 0.17 \\
\hline Family 19. Diadesmidaceae & 3 & 6 & 7 & 1.24 \\
\hline Family 20. Diploneidaceae & 1 & 6 & 6 & 1.06 \\
\hline Family 21. Naviculaceae & 6 & 55 & 68 & 12.01 \\
\hline Family 22. Naviculales incertae sedis & 3 & 4 & 4 & 0.71 \\
\hline Family 23. Neidiaceae & 2 & 7 & 9 & 1.59 \\
\hline
\end{tabular}




\begin{tabular}{|c|c|c|c|c|}
\hline Family 24. Pinnulariaceae & 1 & 34 & 40 & 7.12 \\
\hline Family 25. Sellaphoraceae & 2 & 6 & 6 & 1.06 \\
\hline Family 26. Stauroneidaceae & 3 & 12 & 14 & 2.48 \\
\hline Order 9. Rhopalodiales & 2 & 9 & 21 & 3.73 \\
\hline Family 27. Rhopalodiaceae & 2 & 9 & 21 & 3.73 \\
\hline Order 10. Surirellales & 5 & 16 & 19 & 3.37 \\
\hline Family 28. Entomoneidaceae & 1 & 3 & 4 & 0.71 \\
\hline Family 29. Surirellaceae & 4 & 13 & 15 & 2.66 \\
\hline Order 11. Tabellariales & 4 & 10 & 13 & 2.32 \\
\hline Family 30. Tabellareaceae & 4 & 10 & 13 & 2.32 \\
\hline Order 12. Thalassiophysales & 1 & 8 & 12 & 2.13 \\
\hline Family 31. Catenulaceae & 1 & 8 & 12 & 2.13 \\
\hline Class 2. Coscinodiscophyceae & 2 & 15 & 16 & 2.84 \\
\hline Order 13. Aulacoseirales & 1 & 6 & 7 & 1.24 \\
\hline Family 32. Aulacosiraceae & 1 & 6 & 7 & 1.24 \\
\hline Order 14. Coscinodiscales & 1 & 8 & 8 & 1.43 \\
\hline Family 33. Coscinodiscaceae & 1 & 8 & 8 & 1.43 \\
\hline Order 15. Melosirales & 1 & 1 & 1 & 0.17 \\
\hline Family 34. Paraliaceae & 1 & 1 & 1 & 0.17 \\
\hline Class 3. Mediophyceae & 3 & 6 & 6 & 1.06 \\
\hline Order 16. Stephanodiscales & 3 & 6 & 6 & 1.06 \\
\hline Family 35. Stephanodiscaceae & 3 & 6 & 6 & 1.06 \\
\hline $\begin{array}{l}\text { Class 4. Bacillariophyta classis } \\
\text { incertae sedis }\end{array}$ & 2 & 2 & 2 & 0.35 \\
\hline $\begin{array}{l}\text { Order } 17 . \text { Bacillariophyta ordo } \\
\text { incertae sedis }\end{array}$ & 2 & 2 & 2 & 0.35 \\
\hline $\begin{array}{l}\text { Family 36. Bacillariophyta familia } \\
\text { incertae sedis }\end{array}$ & 2 & 2 & 2 & 0.35 \\
\hline
\end{tabular}

\section{Conclusion}

The unique, low studied localities of diatom algae - Pamir mountain aquatic habitats included 455 species (552 with infraspecific taxa) of diatoms that we here presented in the first time as a result of references data of 19302000 compilation with the species list from our study in 2000-2015. Systematical structure analysis revealed Class Bacillariophyceae, Order Naviculales, and Genus Nitzschia as most richest. The Pinnularia genus with extremely large species richness, 39, show peculiarities of the Pamir diatom 
flora because prefer fresh, clear, circumneutral waters in natural aquatic objects with developed phytoperiphytonic communities, included many rare species. Comparison of revealed diatom species richness in Pamir with the surrounding mountain algal floras let us to assume that total algal flora of Pamir can reach about 1,000 taxa.

\section{Acknowledgements}

This work was partly funded by the Israeli Ministry of Absorption.

\section{References:}

1. Balashova, N.B., Nikitina, V.N. (1978). To the algal flora of some thermal springs of Pamir. Proceedings of VI conference of lower plants of Middle Asia and Kazakhstan. Donish, Dushanbe, p. 22.

2. Aysel, V. (2005). Check-list of the freshwater algae of Turkey. Journal of the Black Sea/Mediterranean Environment, 11, 1-124.

3. Barinova, S, Naiz Ali, Barkatullah, Sarim, F.M. (2013). Ecological Adaptation to Altitude of Algal Communities in the Swat Valley (Hindu Cush Mountains, Pakistan). Expert Opinion on Environmental Biology, 2(2), 1-15, DOI: 10.2478/s13545-014-0150-y

4. Barinova, S. (2011). Algal Diversity Dynamics, Ecological Assessment, and Monitoring in the River Ecosystems of the Eastern Mediterranean. Hauppauge, NY, USA: Nova Science Publishers, 363 p.

5. Barinova, S., Boboev, M., Hisoriev, H. (2015a). Freshwater algal diversity of the South-Tajik Depression in a high mountainous extreme environment. Turkish Journal of Botany, 39, 535-546, and Supplement 1-22, doi:10.3906/bot-1406-45.

6. Barinova, S., Gabyshev, V., Boboev, M., Kukhaleishvili, L., Bilous, O. (2015b). Algal Indication of Climatic Gradients, American Journal of Environmental Protection. Special Issue: Applied Ecology: Problems, Innovations, 4(3-1), 72-77, doi: 10.11648/j.ajep.s.2015040301.22.

7. Barinova, S., Kukhaleishvili, L. (2014). Diversity and ecology of algae and cyanobacteria in the Aragvi River, Georgia. The Journal of Biodiversity, Photon, 113, 305-338.

8. Barinova, S., Niyatbekov, T.P. (2017). Algal Diversity of the Pamir High Mountain Mineral Springs in Environmental Variables Gradient. International Journal of Environmental Sciences \& Natural Resources, 7(2): 555706. DOI: 10.19080/IJESNR.2017.07.555706.

9. Barinova, S.S., Kukhaleishvili, L., Nevo, E., Janelidze, Z. (2011). Diversity and ecology of algae in the Algeti National Park as a part of the Georgian system of protected areas. Turkish Journal of Botany, 35, 729-774. DOI 10.3906/bot-1009-83. 
10. Baturina, L.R. (1983). Algae of thermal springs of Tajikistan. $\mathrm{PhD}$ Thesis, Dushanbe, 284 p.

11. Bokhodjaev, I.Ya., Davlatmamadov, Sh.M. (1994). Medical mineral springs of Pamir. Dushanbe.

12. Churshina, N.M. (1982). Mineral, thermal and cold water. In: Tajikistan (nature and natural resources). Dushanbe, pp. 125-131.

13. Ergashev, A.E. (1969). Materials to the algal floras of natural and artificial, thermal and hot springs of Middle Asia. Lower Plants of Middle Asia. Tashkent, Fan, 3-37.

14. Jumaeva, G.R. (2008). Algal flora of major thermal and mineral springs of Pamir. PhD Thesis, Dushanbe, $151 \mathrm{p}$.

15. Muzafarov, A.M. (1965). Algal flora of the Central Asian waterbodies. Tashkent, Uzbekistan Academy of Science Publisher.

16. Petersen, J.B. (1930). Algae from O. Olufsen's second Danish Pamir Expedition 1898-1899. Dansk Bot. Ark., 6(6), 1-60.

17. Stevenson, J. (2014). Ecological assessments with algae: a review and synthesis. Journal of Phycology, 50, 437-461, doi:10.1111/jpy.12

18. Zarei Darki, B. (2009). Taxonomic structure of the algal flora of Iran. Bangladesh Journal of Plant Taxonomy, 16(2), 185-194, DOI: 10.3329/bjpt.v16i2.3933. 